\title{
Ultra Short Pulsed Laser Deposition Technology for Industrial Applications
}

Dr Jari Liimatainen*, Ville Kekkonen, Jarkko Piirto, Juho Kaisto, Dr Aleksey Zolotukhin and Dr Saumyadip Chaudhuri

Picodeon Oy Ltd, Piisilta 1, 91100, Ii, Finland

\begin{abstract}
PLD (Pulsed laser deposition technology) has been actively developed since 1980's for variety of applications and materials systems. PLD technology has been considered as an attractive, flexible production method for complex oxides due to its intrinsic benefits for maintaining stoichiometry and producing good quality thin films e.g. for high temperature superconductors. Stoichiometric material transfer from target to substrate, excellent adhesion for various substrates and possibility to process wide range of materials is attractive technical advantages that can be utilized in variety of existing and emerging application areas. Industrial applications have been limited by e.g. undesirable particle formation in traditional PLD technology based on ns-second pulse lengths and challenges in scale-up for industrial production. This paper describes recent findings in the development and use of ultrashort, ultrafast pulse laser deposition technology for nanoporous functional films using ultra short pulse and ultrashort repetition rate laser and advanced scanning technology.
\end{abstract}

Key words: PLD (Pulsed laser deposition), cold ablation, metallic thin films, sensors, phovoltaics, lithium ion battery coatings.

\section{Introduction}

PLD (Pulse laser deposition) technology is versatile materials processing and thin film manufacturing technology allowing several technological benefits. Possibility for stoichiometric materials transfer, excellent adhesion, flexibility for material selection and possibility to apply coatings on heat sensitive materials are benefits that it has been realized and used in designing thin film solutions based on PLD technology [1].

Even though PLD has been actively studied for wide range of applications, its current industrial applications are limited to e.g high temperature superconductors YBCO (Yttrium barium copper oxide) where utilization of stoichiometric material transfer from target to substrate is essential [2]. However, the intrinsic benefits of PLD offer possibility for much wider portfolio of applications including hard

*Corresponding author: Dr Jari Liimatainen, chief technology officer, research fields: materials science, material processing, thin film technology and nanotechnology. E-mail: Jari.Liimatainen@picodeon.com. tribological surfaces, optical solutions, medical applications, sensors, PZT, corrosion protection layers, metallizing, lithium ion batteries and photovoltaics applications [2-4].

Particle formation has been traditionally a challenge especially in PLD technology utilizing pulse length in nanosecond regime [5]. Droplets caused by thermal ablation, fragments from target surface or nanoparticle formation can be detrimental for thin film integrity or its material properties. Partially, particle formation is related to quality of target materials, partially for finetuning ablation parameters and quality of laser beam.

On the other hand, PLD technology also allows accurate adjustment of nano- and microscale porosity by controlling formation of particulates and subsequently transfer to substrate surface. Control of nanostructure, surface area and nanoscale porosity is essential feature in e.g. lithium ion battery (separator film layers), sensor (high sensitivity sensing layers) and photovoltaics (catalytic surfaces) applications. Roll-to-roll and sheet-to-sheet type of manufacturing 
can be integrated for PLD deposition using suitable scanning techniques allow high productivity.

Objective of this work is to investigate \& demonstrate technology to control structure, porosity and selected technical properties of metallic materials $(\mathrm{Au}, \mathrm{Cu}, \mathrm{W})$ and oxides $\left(\mathrm{Al}_{2} \mathrm{O}_{3}\right.$ and $\left.\mathrm{WO}_{3}\right)$ in ultrashort pulsed lased deposition processing.

\section{Materials and Methods}

\subsection{Materials}

Materials used in the deposition trials are presented in Table 1. Metallic materials gold, copper and tungsten as well as inorganic ceramic materials tungsten trioxide and aluminium oxide were investigated for their behavior in ultrashort pulsed laser deposition.

\subsection{PLD Deposition Methods}

PLD deposition experiments were carried out using Picodeon Series 2 and Series 4 PLD deposition tools, see Fig. 1 and Table 2. Series 2 PLD tool possesses LumeraHyperRapid 50 laser with maximum average power of $50 \mathrm{~W}$, repetition rates up to $1 \mathrm{MHz}$, pulse length 9 ps and galvanometric scanner. Series 4 PLD tool utilizes Amphos Flex 150 laser with repetition rates up to $0.7-40.5 \mathrm{MHz}$, pulse length of $0.65-9$ picosecond and polygonal scanning followed by

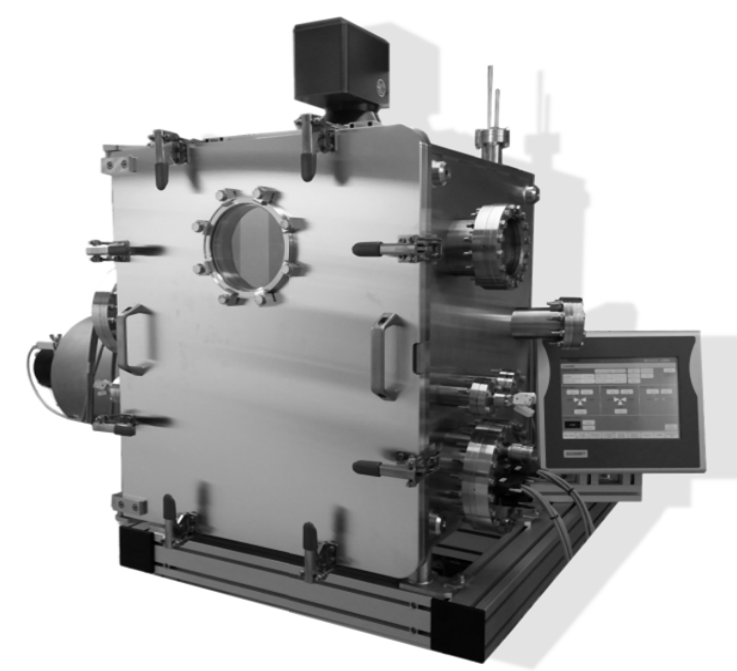

(a) telecentric lens. Deposition key parameters used in the experiments are presented in Table 3.

\subsection{In-Situ Process Metrology}

Process conditions were investigated during deposition trials using optical emission spectroscopy, quartz crystal balance and temperature measurements of target and substrate. Used testing equipments were presented in Table 4 and Fig. 2.

\subsection{Thin Film Characterizations}

Deposited films where characterized using various methods depending on the materials. Filmetrics F20 Spectral Reflectometer thin film analyser was used for thickness and optical analyser. Atomic force microscope NanoforceEasyscan 2 was used for surface

Table 1 Materials used as targets and substrates in deposition experiments.

\begin{tabular}{ll}
\hline Target materials & \\
\hline Metallic & \\
\hline Material & Description \\
Cu & $99.9 \% \mathrm{Cu}$, hot rolled \\
$\mathrm{Au}$ & $99.9 \% \mathrm{Au}$, rolled $\mathrm{Au}$ \\
$\mathrm{W}$ & $99.9 \%$ Sintered target \\
\hline Ceramic & \\
\hline Aluminium oxide & Sintered target \\
Aluminium oxide & Sintered target \\
Tungsten trioxide & Sintered target \\
\hline
\end{tabular}

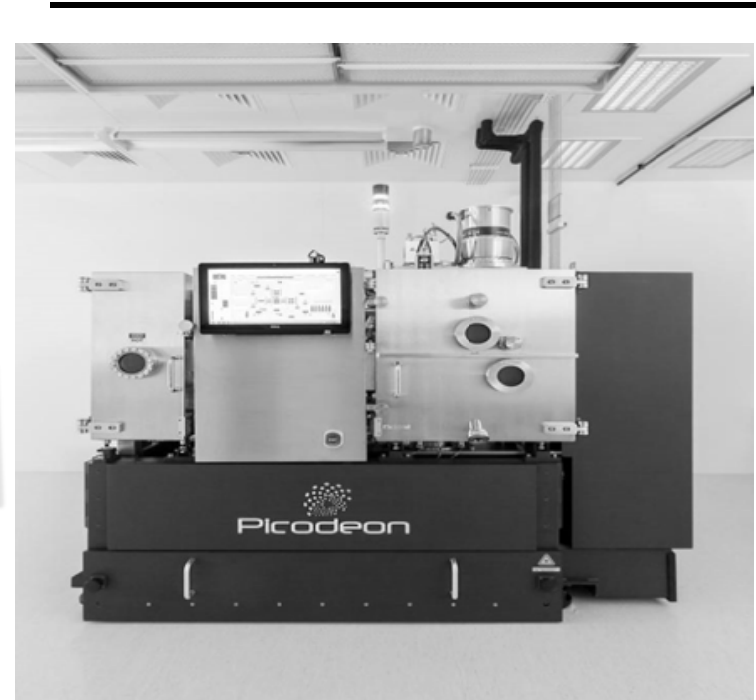

(b)

Fig. 1 PLD equipments used in deposition experiments: (a) Series 2 and (b) Series 4. 
Table 2 Key features of PLD equipments used in the deposition trials.

\begin{tabular}{llllll}
\hline & Average power & Repetition rate & Pulse length & Wavelength & Scanning \\
\cline { 2 - 6 } & $\mathrm{W}$ & $\mathrm{MHz}$ & Picoseond & $\mathrm{nm}$ & \\
\hline Series2 & 50 & 1 & 9 & 355 & Galvanometric \\
Series4 & 200 & $0.7-40.5$ & $0.65-9$ & 1063 & Polygonal \\
\hline
\end{tabular}

Table 3 Deposition parameters used.

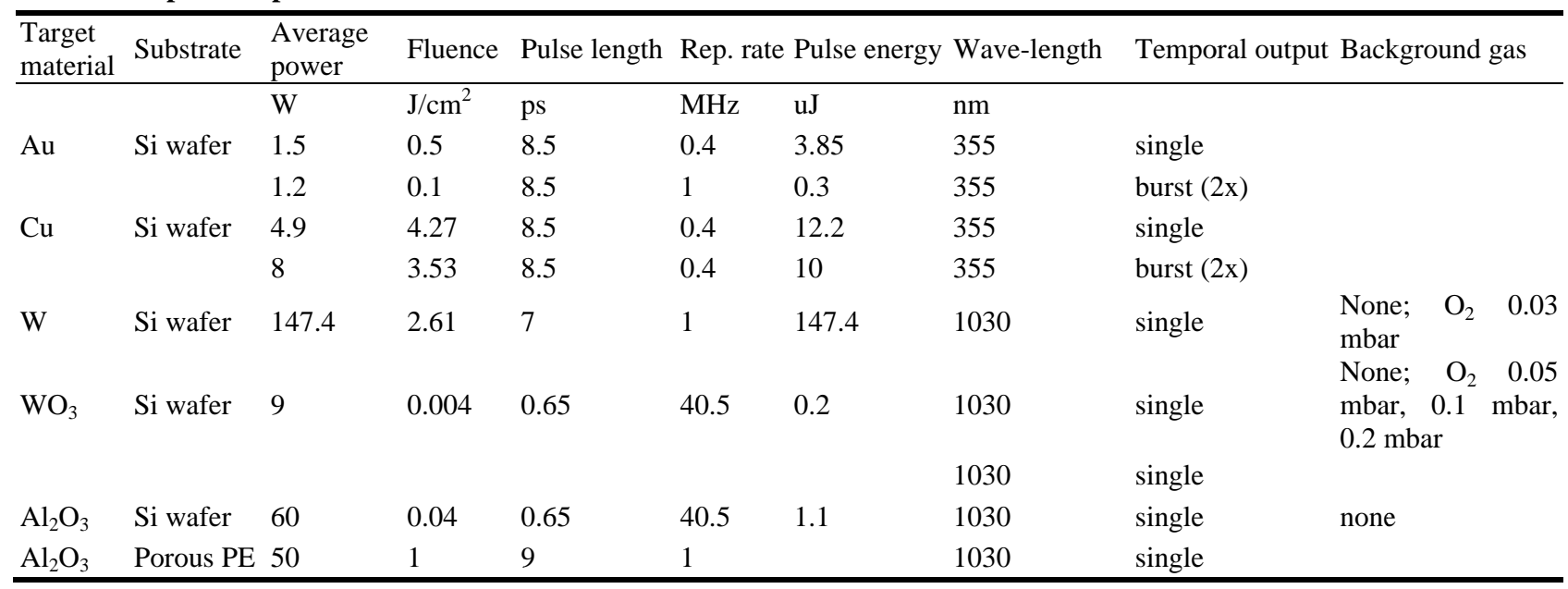

Table 4 In-situ process metrology used in deposition experiments.

\begin{tabular}{|l|l|l|}
\hline Methods & Equipments & Testing purposes \\
\hline Quartz crystal balance & Inficon quartz crystal microbalance & $\begin{array}{l}\text { Materials transfer and particle formation during } \\
\text { deposition }\end{array}$ \\
\hline Optical emission spectroscopy & Ocean optics spectrometer & Plume ionization \\
\hline Temperature measurements & Thermocouples & $\begin{array}{l}\text { Temperature control of target and substrate during } \\
\text { deposition }\end{array}$ \\
\hline
\end{tabular}

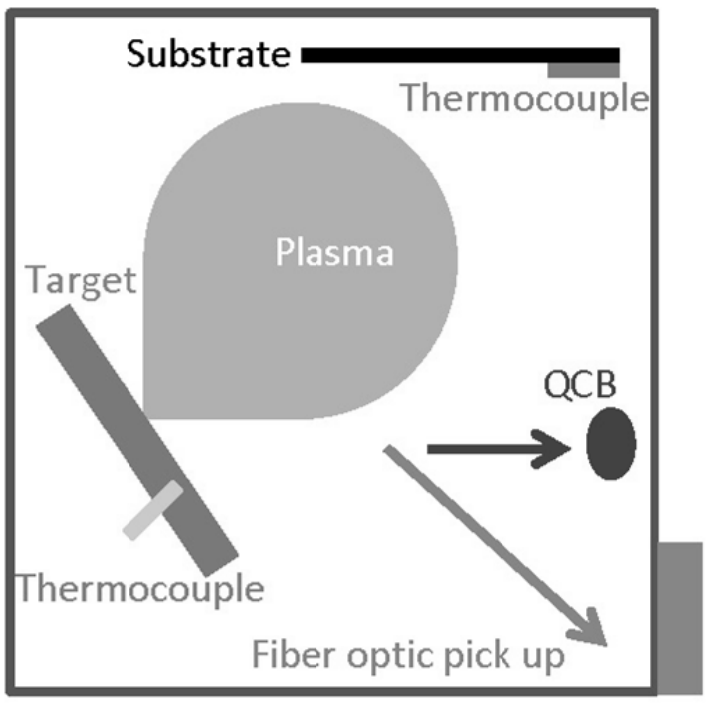

Fig. 2 Optical emission spectroscopy measurement arrangement.

topography investigations. Scanning electron microscopy Zeiss Sigma \& UltraPlus was used to characterize microstructures and nanoparticles.

\section{Results}

\subsection{In-Situ Process Metrology}

\subsubsection{Temperature Measurements}

During the deposition of copper and aluminium oxide on silicon, in-situ measurement of the silicon temperature was carried out using k-type thermocouple. The thermocouple was mounted on the substrate side facing the incident plasma and was protected from the plasma by Kapton tape. Additionally, a second thermocouple was inserted into the bulk of the copper target, such that the distance of the thermocouple from the ablation surface was about $0.5 \mathrm{~mm}$. Fig. 3a shows the time evolution of the temperature of the silicon substrate as well as the copper target during deposition. Fig. $3 b$ shows the time evolution of the temperature of the silicon substrate for various power levels of the incident laser during deposition of aluminium oxide. 


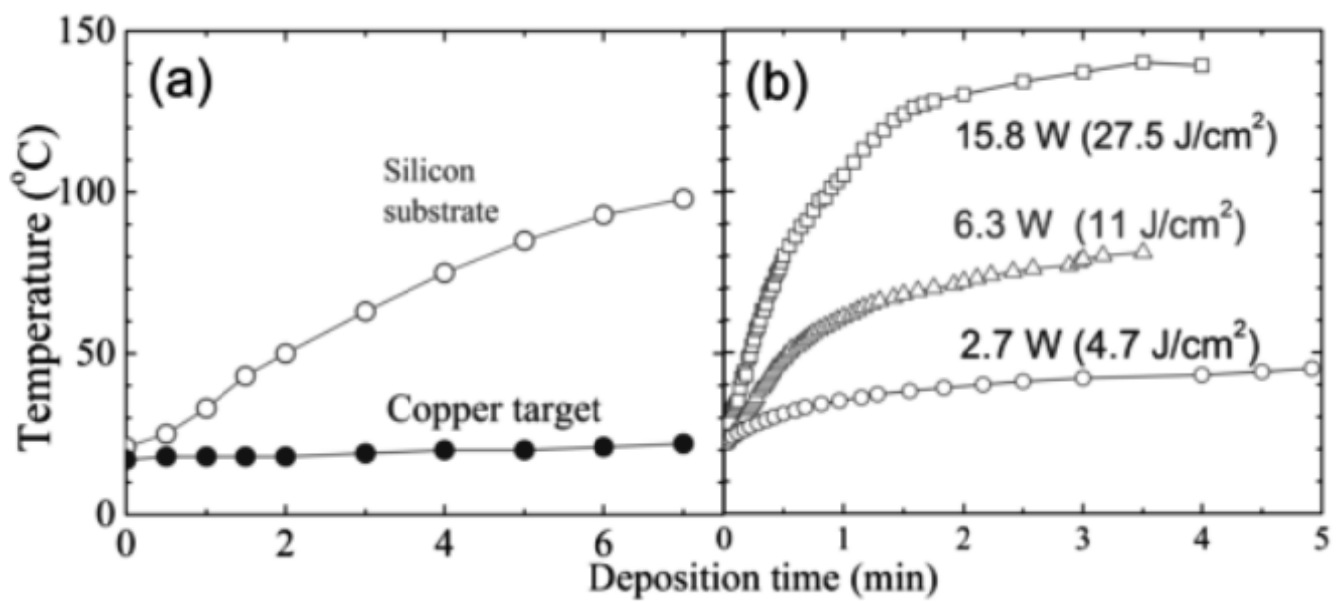

Fig. 3 (a) Time evolution of target and substrate temperatures during the deposition of copper on silicon wafer; (b) Time evolution of the temperature of silicon substrates for various levels laser power incident on an aluminium oxide target. The corresponding values of fluence are quoted in the parenthesis.

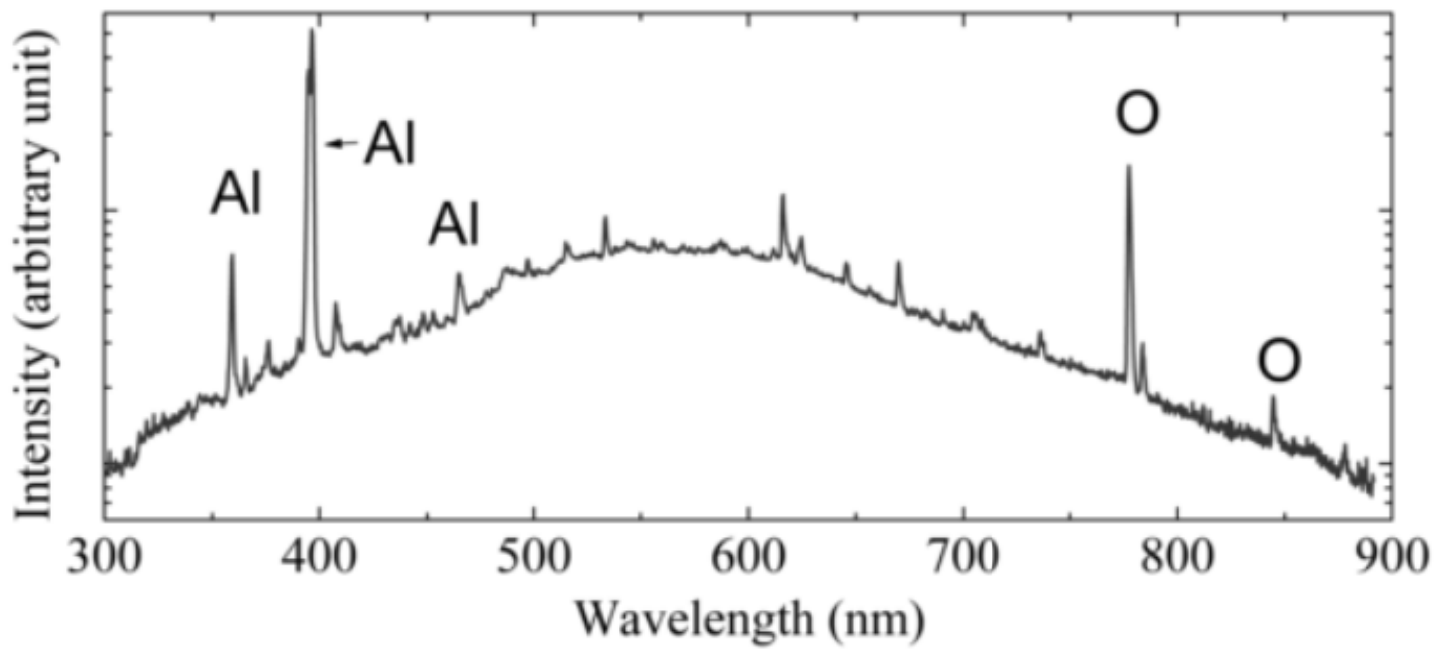

Fig. 4 Optical emission spectra obtained during the ablation of an aluminium oxide target in presence of background oxygen pressure. The electronic transitions corresponding to aluminum and oxygen ions are marked.

\subsubsection{Optical Emission Spectroscopy (OES)}

Fig. 4 shows a typical optical emission spectra obtained during the ablation of an aluminum oxide target in presence of a background oxygen gas. Electronic transitions corresponding to aluminum and oxygen ions are clearly visible.

\subsection{Film Structures}

\subsubsection{Metallic Films}

Deposited metallic film microstructures are presented in Fig. 5. Metallic gold and copper films were produced both at various porosity levels by adjustment of PLD processing parameters and background gas. Tungsten depositions were produced as porous films. Density of gold and copper films was modified adjusting fluence and temporal laser output by applying burst mode.

In the case of tungsten, Fig. 7, background gas pressure was used as an additional process parameter in order to promote particulate formation. Thin film density with $10^{-6}$ mbar $\mathrm{O}_{2}$ background gas was more dense without microporosity. Without use of background 


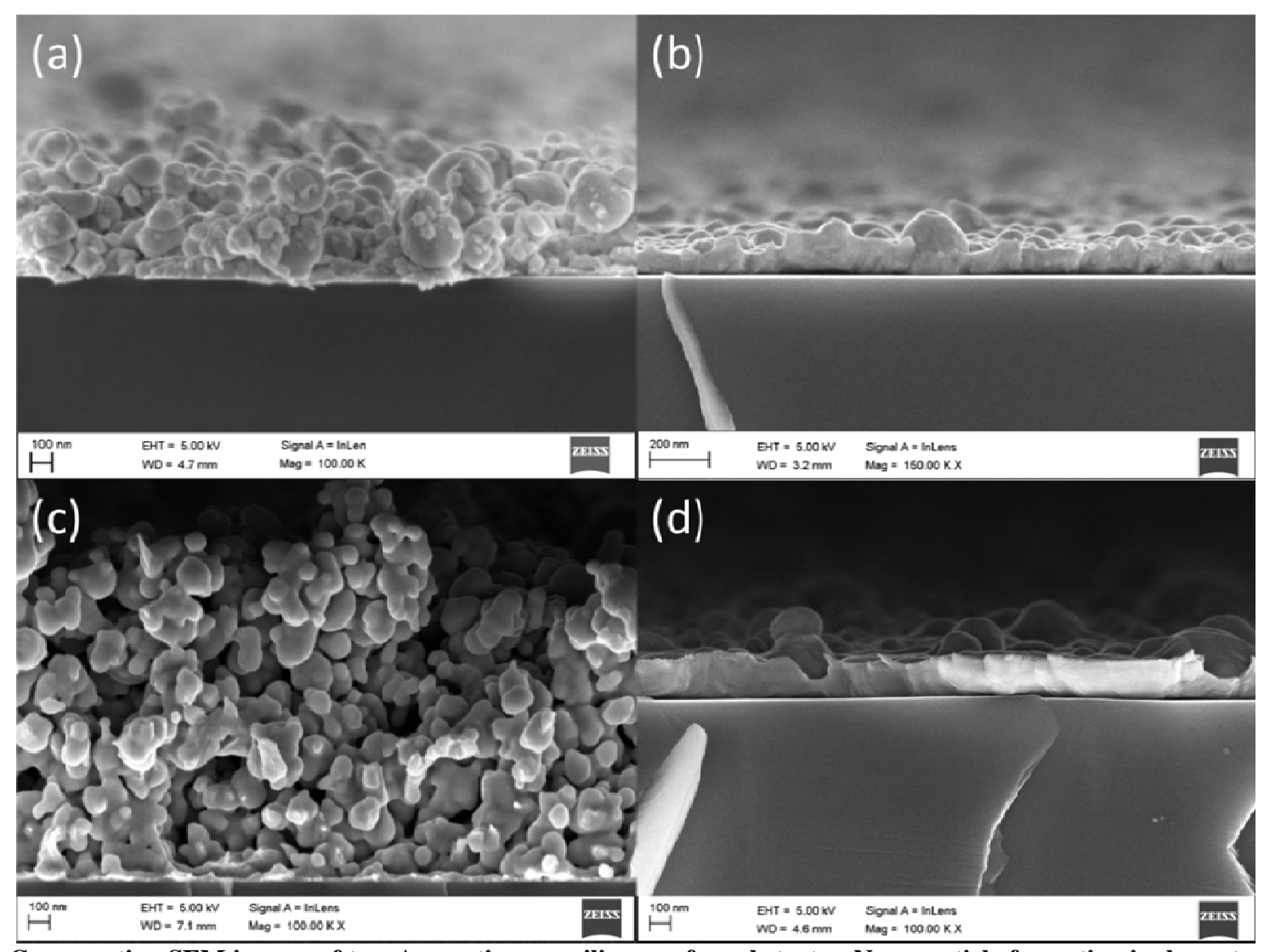

Fig. 5 Cross-section SEM images of two Au coatings on silicon wafer substrates. Nanoparticle formation is characteristic to single pulse ablation mode (a). Significant reduction of particles can be obtained through double-pulse ablation mode which also leads to increase in density of the deposited layer (b). The same phenomenon applies also to pulsed laser deposition of $\mathrm{Cu}$ layers (c), (d).
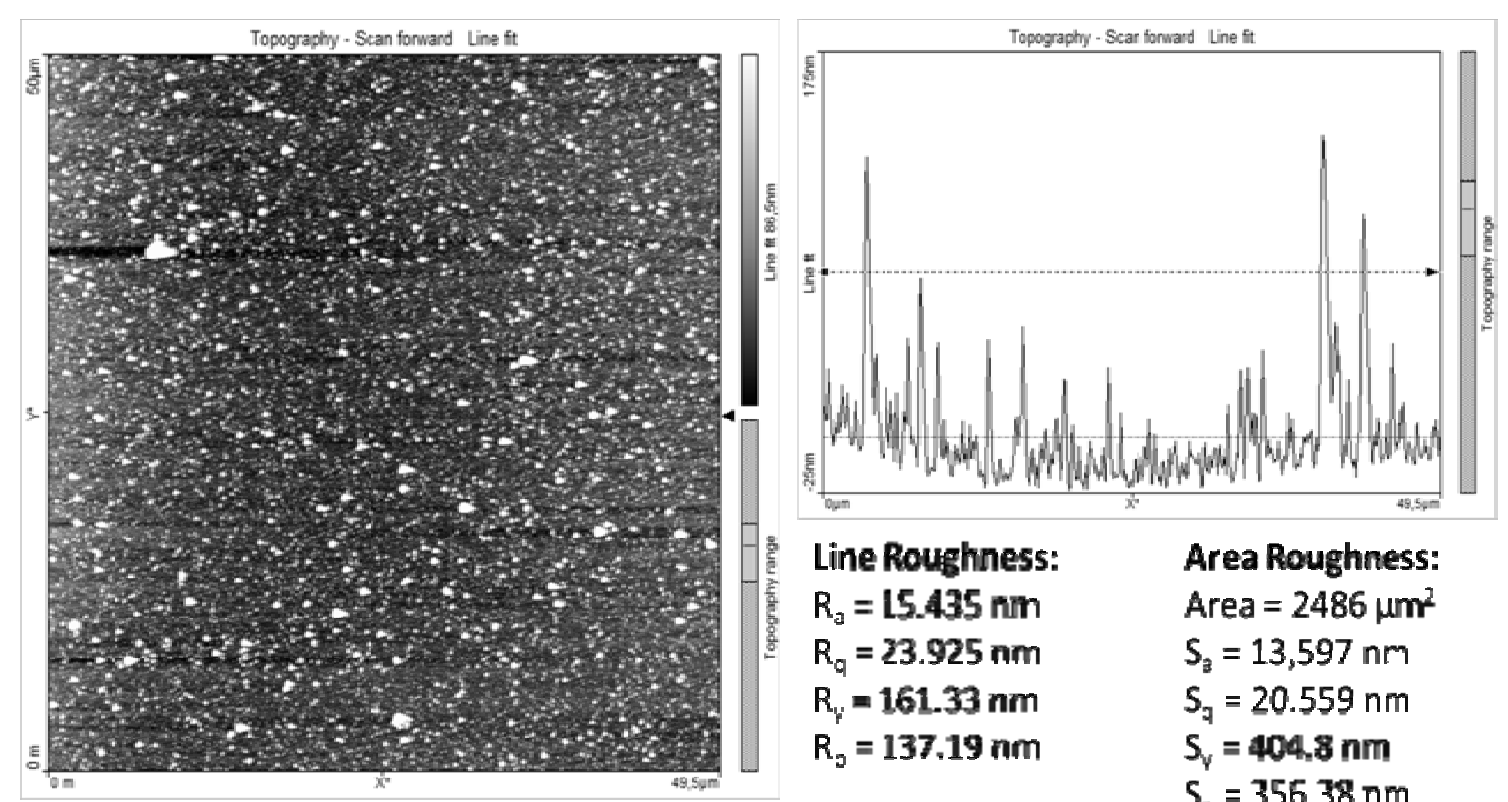

$$
\begin{array}{ll}
\text { Line Roughnes: } & \text { Area Roughness: } \\
R_{\mathrm{a}}=15.435 \mathrm{~nm} & \text { Area }=2486 \mu \mathrm{m}^{2} \\
R_{\mathrm{q}}=23.925 \mathrm{~nm} & S_{\mathrm{a}}=13,597 \mathrm{~nm} \\
R_{\mathrm{p}}=161.33 \mathrm{~nm} & S_{\mathrm{q}}=20.559 \mathrm{~nm} \\
R_{0}=137.19 \mathrm{~nm} & S_{\mathrm{V}}=404.8 \mathrm{~nm} \\
& S_{\mathrm{p}}=356.38 \mathrm{~nm}
\end{array}
$$

\section{Area Roughness:}

Fig. 6 AFM measurement results of Au coating were deposited utilizing the double-pulse ablation mode. 


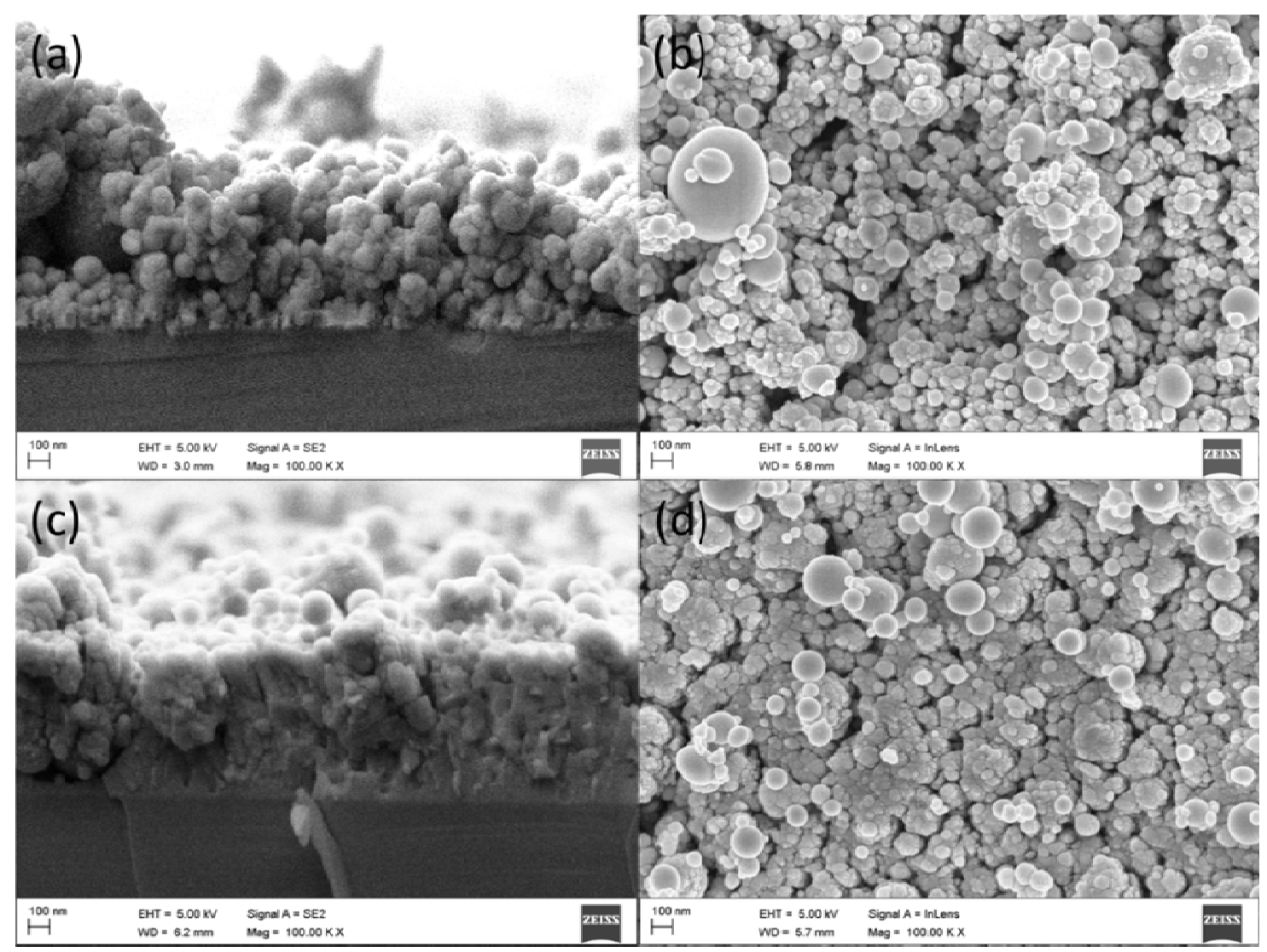

(a)

(b)

Fig. 7 (a) Cross-section and (b) surface SEM images of a $W$ coating on silicon wafer substrate deposited in vacuum show presence of spherical particles mostly in size range of $10-200 \mathbf{n m}$. Using otherwise same parameters but adding $\mathrm{O}_{2}$ background gas to a deposition pressure of $3.1 \times 10^{-2}$ mbar results in significant densification most probably due to oxidation process.

gas it was possible to produce tungsten thin film structure with porosity and particulated material layers were clearly visible on substrate surface. Particulate shape was mainly spherical with size of 10-200 nm.

3.2.2 Aluminium Oxide and Tungsten Trioxide Films

For aluminium oxide films laser fluence, repetition rate, pulse length and energy as well as use of background gas were adjusted to modify the nanostructure and porosity of films, see Figs. 8a and 8b. At low fluences $0.04 \mathrm{~J} / \mathrm{cm}^{2}$, high repetition rates (40.5 MHz), short pulse length (0.65 ps) and low pulse energies (1.1 uJ) dense film were created. Using higher fluences $\left(1 \mathrm{~J} / \mathrm{cm}^{2}\right)$, lower repetition rates (0.7 MHz), longer pulses (9 ps) and $\mathrm{O}_{2}$ background gas much more particulate based material transfer was created. Particulate shape was mixed ranging from mainly spherical to globular and irregular shapes. Irregular shaped particles are possibly result of fragment formation in the target surface during ablation and their shape is smoothened by partially atomized/ionized material in the plume.

For tungsten trioxide films effect of background gas on micro- and nanostructure was significant. Without background gas tungsten trioxide film was dense (Figs. 9a and 9b) while increasing background gas increased porosity according to Fig. 9c-9h.

\subsection{Film Uniformity}

Film thickness uniformity was investigated for dense $\mathrm{Al}_{2} \mathrm{O}_{3}$ films producedusing real time scan line laser energy control and wafer rotation. Thickness non-uniformity levels of $<1.5 \%$ were achieved for $100 \mathrm{~mm}$ silicon wafers. 

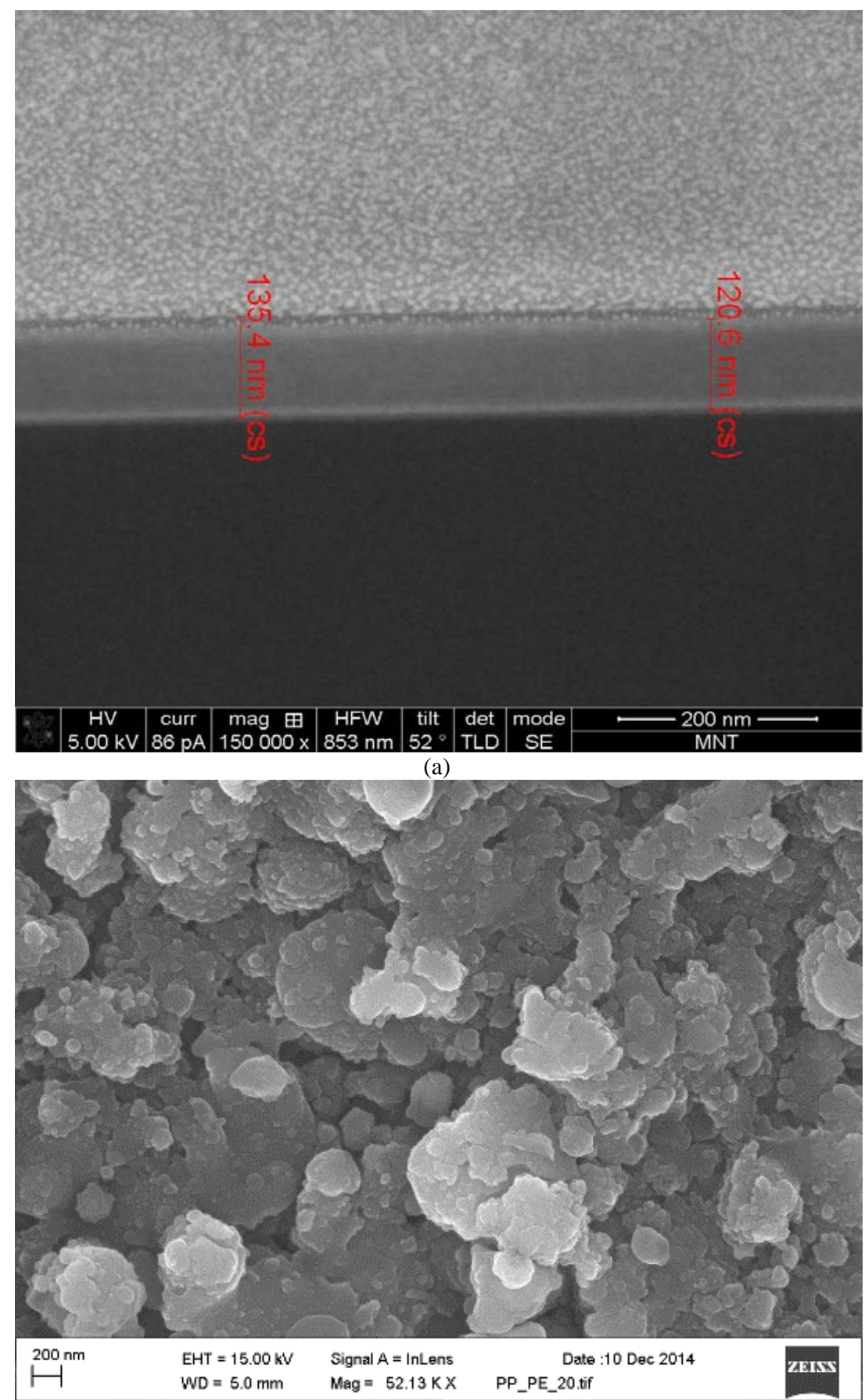

(b)

Fig. 8 Aluminium oxide films by SEM. (a) Dense films produced at high repetition rates (40.5 MHz), short pulse (0.65 ps), low fluence $\left(0.04 \mathrm{~J} / \mathrm{cm}^{2}\right)$ and (b) porous films produced using low repetition rate (1 MHz), long pulse $(9 \mathrm{ps})$, high fluence $\left(1 \mathrm{~J} / \mathrm{cm}^{2}\right)$ and background gas.

\subsection{Adhesion of Thin Films}

Adhesion test results are presented in Table 5 . Aluminium oxide films where tested both as deposited on silicon wafers and on special, and porous polyethylene membrane is used in lithium ion battery separator film applications. Thin films produced on polyethylene separator film were tested using pull testing and severe bending testing.

Copper adhesion to silicon without interim glue layers 


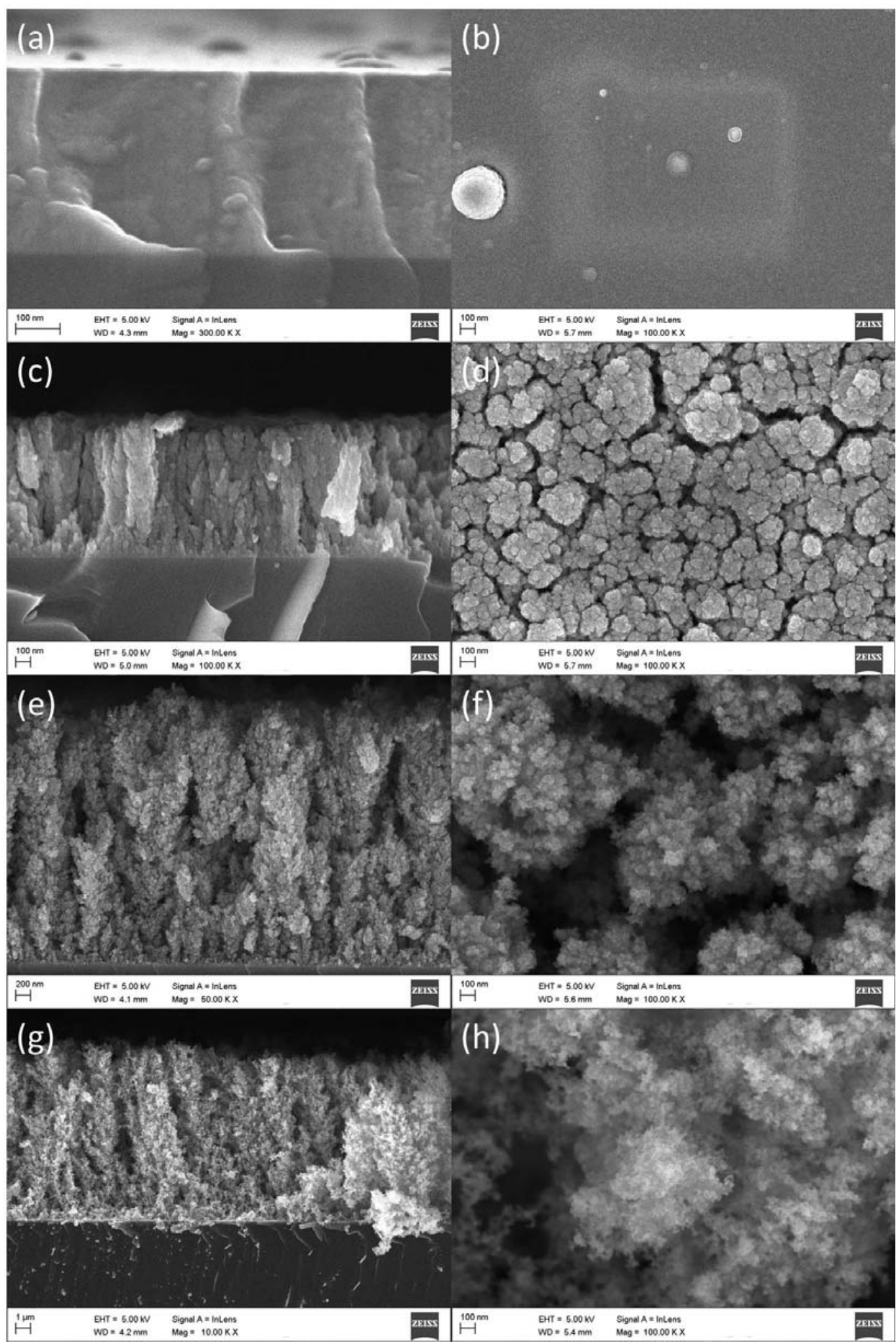

Fig. 9 A series of SEM images demonstrate the formation of nanostructures and porosity with the use background gas in the deposition process of $\mathrm{WO}_{3}$. Same deposition parameters are used for all of the four samples presented in the images except for the background gas $\left(\mathrm{O}_{2}\right)$ pressure: (a-b) no background gas, (c-d) 0.05 mbar, (e-f) 0.1 mbar and (g-h) 0.2 mbar background gas. 
Table 5 Adhesion test results for deposited films using pull test.

\begin{tabular}{llll}
\hline Target material & Substrate & Bond strength & Note \\
\hline $\mathrm{Cu}$ & & $\mathrm{N} / \mathrm{mm}^{2}$ & \\
$\mathrm{Al}_{2} \mathrm{O}_{3}$ & Si wafer & $>40$ & Silicon wafer broke \\
$\mathrm{Al}_{2} \mathrm{O}_{3}$ & Si wafer & $>40$ & Silicon wafer broke \\
$\mathrm{Al}_{2} \mathrm{O}_{3}$ & Polyethylene & $1.5-2$ & \\
\hline
\end{tabular}

was tested using pull testing. In most cases silicon wafer cracked before debonding of the copper layer from silicon. Same results were obtained for dense $\mathrm{Al}_{2} \mathrm{O}_{3}$ deposited films i.e. silicon wafers cracked before $\mathrm{Al}_{2} \mathrm{O}_{3}$ layer debonded from silicon wafers. Porous $\mathrm{Al}_{2} \mathrm{O}_{3}$ deposited on porous polyethylene membrane yielded $>50 \%$ higher adhesion as compared to slurry coated $\mathrm{Al}_{2} \mathrm{O}_{3}$ layer with polymer binder.

\section{Discussion}

The process metrology based on target temperature measurements indicates that the ablation process in case of copper has been carried out in so called cold ablation regime. Temperature increase of copper target after several minutes of ablation was $<10^{\circ} \mathrm{C}$. However, it is obvious from characterization of deposited films that some cluster and particle formation occurs after initial ablation in the atomized plume. The role of droplet formation from target surface cannot be excluded, but moderate temperature increase after several minutes of ablation proposes that its role has been small. The double formation in pulsed laser deposition of metals is complex phenomen various mechanism are involved. Droplet formation has been investigated by atomistic computer simulations by Zhigilei et al. [6].

Use of burst i.e. double pulse increases density of deposited copper films and can be consequently used to adjust the film density and inter-particle adhesion. The role of temporal output of laser pulses has been investigated for e.g. $\mathrm{LaAlO}_{3}$, and it proposed that the plasma absorption and following re-evaporation may explain its impact on increased film density [7]. This finding is in accordance with results of this study both for copper and gold depositions. The use of secondary laser beam to reduce particle density of deposited film has been successfully experimented by György et al. [8].

As the burst mode should impact the initial stages of plume formation this means that particles are formed and partially subsequently atomized as free particles close to target surface. Cluster and particle formation occurs soon after ablation in supersaturated plume. Control of the relative shares of atomized material and particles is essential technical feature in engineering the nanoporosity of the films as well as in developing adhesion to substrate and interparticle adhesion i.e. overall film mechanical integrity. For copper and and gold the use of burst mode of pulse shape and optimum fluence increases the share of dense film and can be used to adjust the density as well as bonding between particles and with the substrate.

In the case of tungsten with a target to maximize particle formation, the use of background gas, against expectations, reduced porosity while deposition at $10^{-6}$ mbar vacuum created targeted porous structures from metallic tungsten particles of various size. The reason for this phenomenom is under review.

For aluminium oxide films deposited using high repetition rate $(40.5 \mathrm{MHz})$, short pulse length $(0.65 \mathrm{ps})$, low fluence of $\left(0.04 \mathrm{~J} / \mathrm{cm}^{2}\right)$ and pulse energy $(1.1 \mathrm{uJ})$ created uniform, dense film with reasonably good optical transparency. Use of lower repetition rate $(0.7 \mathrm{MHz})$, longer pulse (9 ps), higher fluence $\left(1 \mathrm{~J} / \mathrm{cm}^{2}\right)$ and background gas resulted in coarse film with porosity levels up to $>40 \%$. The film growth mechanism is most probably combination of particle and cluster build up together with condensation of atomized material. Optical electron spectroscopy indicates the existence of ionized plume but thin film structures proposes significant role of particulates in film build-up. 
Particulate formation seems to be result of various mechanisms including fragments, droplets and particle re-cluster from the plasma as well as condensation of ionized material. The share of these mechanisms was not possible to determine. At high repetition rate and low pulse energy depositions there is now signs of ionized species in the plume based on optical electron microscopy. At low fluence version of $\mathrm{Al}_{2} \mathrm{O}_{3}$ deposition optical electron spectroscopy indicates lack of ionization.

Porosity control is essential feature in developing coatings for sensors, catalytic applications and lithium ion battery applications where e.g. active surface control or permeability are essential for the functionality. Additionally, it is important to control the adhesion for substrate as well as film mechanical properties which controlled e.g. by intra-particle bonding. Based on investigations described in this paper nanostructure and porosity of both metallic and oxide thin films can be adjusted using suitable combination of processing parameters.

Adhesion and film integrity based on tests done is very good indication high reliability in applications and products where thin films are subjected to mechanical or thermal loads and where debonding can be fatal.

\section{Conclusions}

Ultrashort pulse laser deposition can be used to adjust nanostructure and -porosity and open surface area of metallic ( $\mathrm{Au}, \mathrm{Cu}$ and $\mathrm{W}$ ) and inorganic metal oxide $\left(\mathrm{Al}_{2} \mathrm{O}_{3}\right.$ and $\left.\mathrm{WO}_{3}\right)$ thin films.

For metallic materials, adjustment of temporal laser output by use of burst mode of laser pulses and control of fluence reduces open surface areas and increases density.

For tungsten deposition, using of background gas $\left(\mathrm{O}_{2}\right)$ reduces microporosity and open surface area of deposited films, without background gas tungsten films are dense with no microporosity.
For inorganic metal oxides $\left(\mathrm{Al}_{2} \mathrm{O}_{3}\right.$ and $\left.\mathrm{WO}_{3}\right)$, using of background gas $\left(\mathrm{O}_{2}\right.$ and $\left.\mathrm{Ar}\right)$ enhances microporosity and maximizes open surface area of deposited films

Adhesion of deposited nanoporous films is good and sufficient for several engineering applications.

Ultrashort pulsed laser deposition is feasible thin film manufacturing method for producing nano- and microporous metallic and metal oxide films for sensors, lithium ion battery, medical and energy storage applications.

\section{References}

[1] Greer, J. A. 2014. "History and Current Status of Commercial Pulsed Laser Deposition Equipment." Journal of Physics D: Applied Physics 47: 1-10.

[2] Fujioka, H. 2015. Handbook of Crystal Growth. Pulsed Laser Deposition, Elsevier B.V.

[3] Dijkkamp, D., Venkatesan, T., Wu, X. D., Shaheen, S. A., Jisrawi, N. and Min-Lee, Y. H. et al. 1987. "Preparation of $\mathrm{Y}-\mathrm{Ba}-\mathrm{Cu}$ Oxide Superconductor This Films Using Pulsed Lase Deposition.” Appied Physics Letters 51: 619.

[4] Lowndes, D. H., Geohegan, D. B., Puretzky, A. A., Norton, D. P. and Rouleau, C. M. 1996. "Synthesis of Novel Thin-Film Materials by Pulsed Laser Deposition.” Science 273: 892-902.

[5] Gamaly, E., Rode, A. and Luther-Davies, B. 1987. "Ultra Fast Laser Ablation and Film Deposition.” R. Eason (Ed), Applications-Led Growth of Functional Materials, John Wiley \& Sons Inc.

[6] Zhigilei, L. V., Zhibin, L. and Ivanov, D. S. 2009. "Atomistic Modeling of Short Pulse Laser Ablation of Metals:Connections between Melting, Spallation and Phase Explosion.” J. Phys. Chem. C 113: 11892-906.

[7] Thelander, E. and Rauscenbach, B. 2012. "Influence of Burst Pulses on the Film Topography in Picosecond Pulsed Laser Deposition of $\mathrm{LaAlO}_{3}$." In proceedings of the 17th International Summer School on Vacuum and Ion Technologies (VEIT 2011), Journal of Physics: Conference Series 356: 1-5.

[8] György, E., Mihailescu, I. N., Kompitsas, M. and Giannoudakos, A. 2004. "Particulates Generation and Solutions For Their Elimination In Pulsed Laser Deposition.” Journal of Optolectronics and Advanced Materials 6 (1): 39-46. 